\title{
Programa saúde da família: a evolução da distribuição espacial das equipes e dos médicos especialistas no Brasil entre 2007 e 2017
}

Lucas Resende de Carvalho, Philipe Scherrer Mendes, Pedro Vasconcelos Maia do Amaral.

\section{RESUMO}

No início da década de 1990, o sistema de saúde brasileiro começou a direcionar esforços para o fortalecimento de uma atenção à saúde preventiva em conjunto com um acompanhamento contínuo das necessidades da população. Para o período de 2007 a 2017, foi observada uma grande expansão do Programa Saúde da Família pelo território brasileiro com significativa interiorização, especialmente das regiões Nordeste, CentroOeste e norte de Minas Gerais. Foi observado ainda um aumento no número de vagas em cursos de graduação de medicina e também um aumento no número de vagas de residência em medicina da família e comunidade, embora com intensa concentração na região Sudeste e em capitais estaduais. Além da formação desses profissionais, destacamos a necessidade da modernização da gestão de recursos humanos para a atração e fixação dos mesmos em áreas de maior necessidade da população, fomentando uma maior equidade do PSF.

Palavras-chave: programa saúde da família, programa mais médicos, distribuição espacial.

Revista da Rede APS 2019 Publicada em: 22/03/2019

Lucas Resende de Carvalho (CEDEPLAR/UFMG),

Philipe Scherrer Mendes (CEDEPLAR/UFMG),

Pedro Vasconcelos Maia do Amaral (CEDEPLAR/UFMG)

Correspondência para:

(Lucas Resende de Carvalho lucasrc@cedeplar.ufmg.b 


\section{INTRODUÇÃO}

O sistema de saúde brasileiro é misto, sendo composto por dois subsistemas, o público - Sistema Único de Saúde (SUS) - e o privado. Com a implementação do SUS em 1988, a Constituição Brasileira passou a garantir o acesso gratuito, universal e integral aos serviços de saúde. A criação do SUS foi o reconhecimento, pela população brasileira, de que o acesso equitativo e igualitário a saúde é um direito fundamental.

O mix público-privado no Brasil se organiza com o SUS ofertando serviços, sejam eles de natureza pública ou por prestadores privados contratados. A característica duplicada da oferta gera uma série de complicações na dinâmica entre os sistemas público e privado de saúde. É característica dos sistemas de saúde duplicados a existência de desigualdades no acesso e, muitas vezes, aqueles que possuem plano de saúde ainda continuam fazendo uso, de alguma maneira, do sistema de saúde público. Segundo Bittencourt (2010) e Borlini (2010), existe também a diferenciação entre a qualidade dos serviços prestados.

Embora a oferta duplicada privada do sistema de saúde ofereça cobertura similar, é verificado, com alguma intensidade, a segmentação na utilização dos serviços em cada sistema. É observado, não somente no Brasil, que o setor público de saúde concentra seu atendimento na baixa e na alta complexidade. Por outro lado, o setor privado foca seu atendimento nos serviços de média complexidade, mais utilizados por aqueles que possuem plano de saúde.

$\mathrm{Na}$ ponta da baixa complexidade, a atenção primária é comumente conhecida como "porta de entrada" para os usuários do sistema de saúde. Seu intuito é o atendimento inicial com o objetivo de filtrar e coordenar o fluxo desse paciente no sistema de saúde.

Os benefícios de um programa de atenção primária eficiente foram elencados por diversas literaturas que mostram uma correlação positiva com a melhora na saúde daquela população. Macinko et al. (2003), por exemplo, mostram que uma atenção primária forte está negativamente associada a mortes por doenças cardiovasculares e respiratórias. Kringos et al. (2013) mostram, para países europeus, que uma atenção primária de qualidade reduz o número de hospitalizações desnecessárias, ocasionando uma redução de custos para o sistema de saúde. O trabalho de Andrade et al. (2015) mostra, para a população de mulheres, gestantes, crianças e idosos do estado de Minas Gerais, que o Programa Saúde da Família é uma política equitativa onde os domicílios mais pobres foram aqueles que apresentaram maiores taxas de visitação. Já Andrade et al. (2017) concluem que o Programa Saúde da Família também é uma política equitativa para a realização do pré-natal.

O objetivo deste trabalho é avaliar a evolução do Programa da Saúde da Família entre 2007 e 2017, em termos territoriais, associando-a ao Programa Mais Médicos (PMM) e aos resultados alcançados na expansão da presença do profissional médico no território nacional. Entende-se que a política implementada em 2013 (PMM) foi de fundamental importância para os avanços obtidos em termos de uma maior cobertura nacional em atenção primária à saúde.

\section{O Programa Saúde da \\ FAMÍLIA NO BRASIL}

No início da década de 1990, o sistema de saúde brasileiro começou a direcionar esforços para o fortalecimento de uma atenção à saúde preventiva em conjunto com um acompanhamento contínuo das necessidades da população. Somado a esses esforços, tem-se um marco importante na consolidação da atenção primária com a criação do Programa Saúde da Família, em 1994, pelo Ministério da Saúde. Segundo Lentsck et al. (2010), a criação do PSF foi fundamental para o começo de uma mudança no modelo assistencial usual hospitalocêntrico, individualizado, de baixa resolutividade e bastante oneroso - em benefício de um modelo que prioriza o cuidado preventivo e contínuo.

A presença do Saúde da Família foi ampliada em todo o território nacional, com uma cobertura de aproximadamente 123 milhões de indivíduos (63\% 


\section{Artigos}

da população brasileira) em 2015, embora sua expansão tenha sido bastante heterogênea entre os municípios (ANDRADE et al., 2018a).

A Figura 1 mostra a expansão da cobertura do Programa Saúde da Família pelo território brasileiro para os anos de 2007 e 2017, evidenciando relevante interiorização da cobertura nos municípios brasileiros, especialmente das regiões Nordeste, Centro-Oeste e norte de Minas Gerais.

Figura 1 - Cobertura populacional do Programa Saúde da Família, por município, Brasil - 2007 e 2017
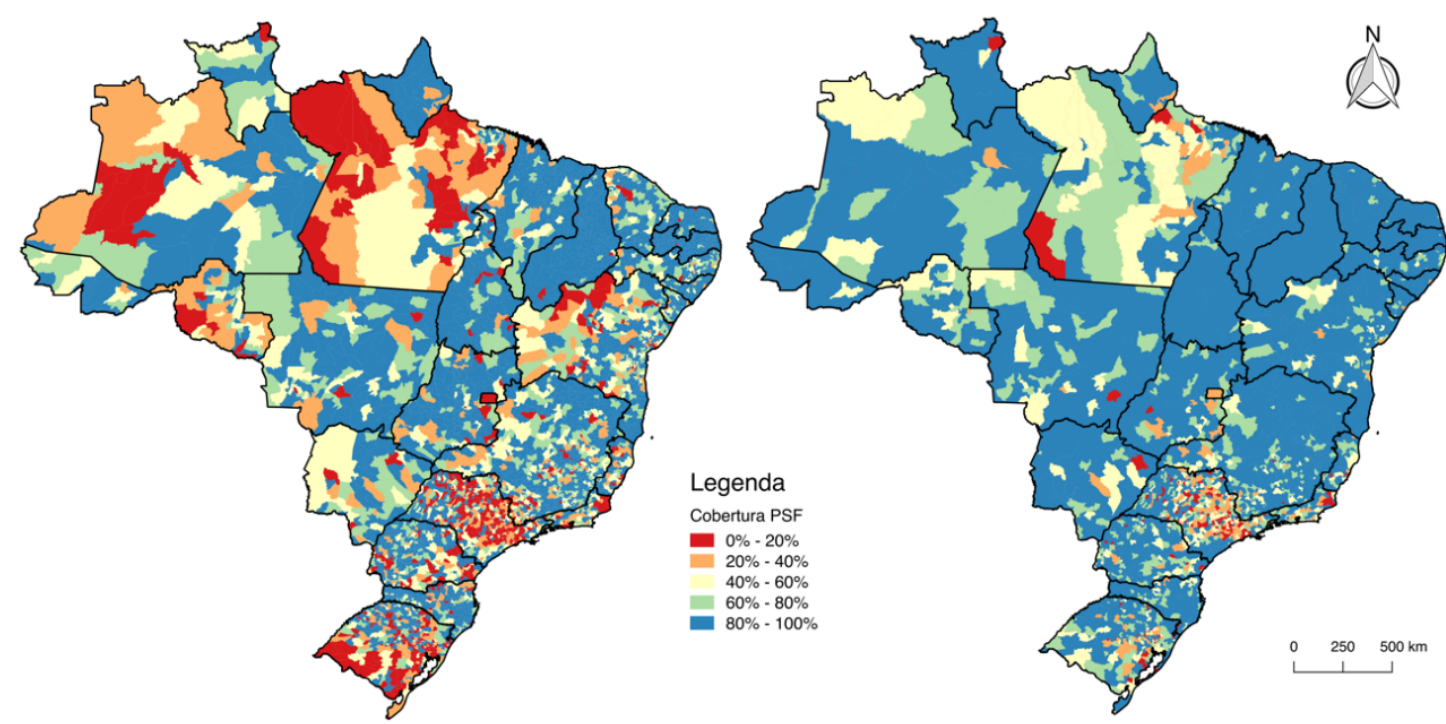

Fonte: Elaboração própria com dados do CNES

A Figura 2 apresenta a composição regional da cobertura municipal do Programa Saúde da Família. Por ela é possível observar que a maioria dos municípios de todas as regiões possui $100 \%$ de cobertura, com o Nordeste chegando a $80 \%$ dos seus municípios sendo integralmente cobertos. As demais macrorregiões possuíam, em 2017, algo em torno de $60 \%$ dos seus municípios com $100 \%$ de cobertura. Além disso, é interessante observar que este percentual cresceu em relação a 2007 e que houve, também, significativa redução dos municípios situados na parte inferior da cauda da distribuição, que possuíam baixa cobertura. Incluise, aí, significativo número de municípios do Norte, Sudeste e Sul que em 2007 possuíam 0\% de cobertura, o que apresenta melhora quando comparado a 2017. Tal comportamento fica evidenciado, também, na Figura 1, em que é possível verificar que, apesar da significativa alteração na representação de 2007 e 2017, as regiões Norte, Sul e Sudeste seguem apresentando maior heterogeneidade na cobertura. 
Figura 2 - Distribuição percentual da cobertura do PSF dos municípios, por região, Brasil - 2007 e 2017

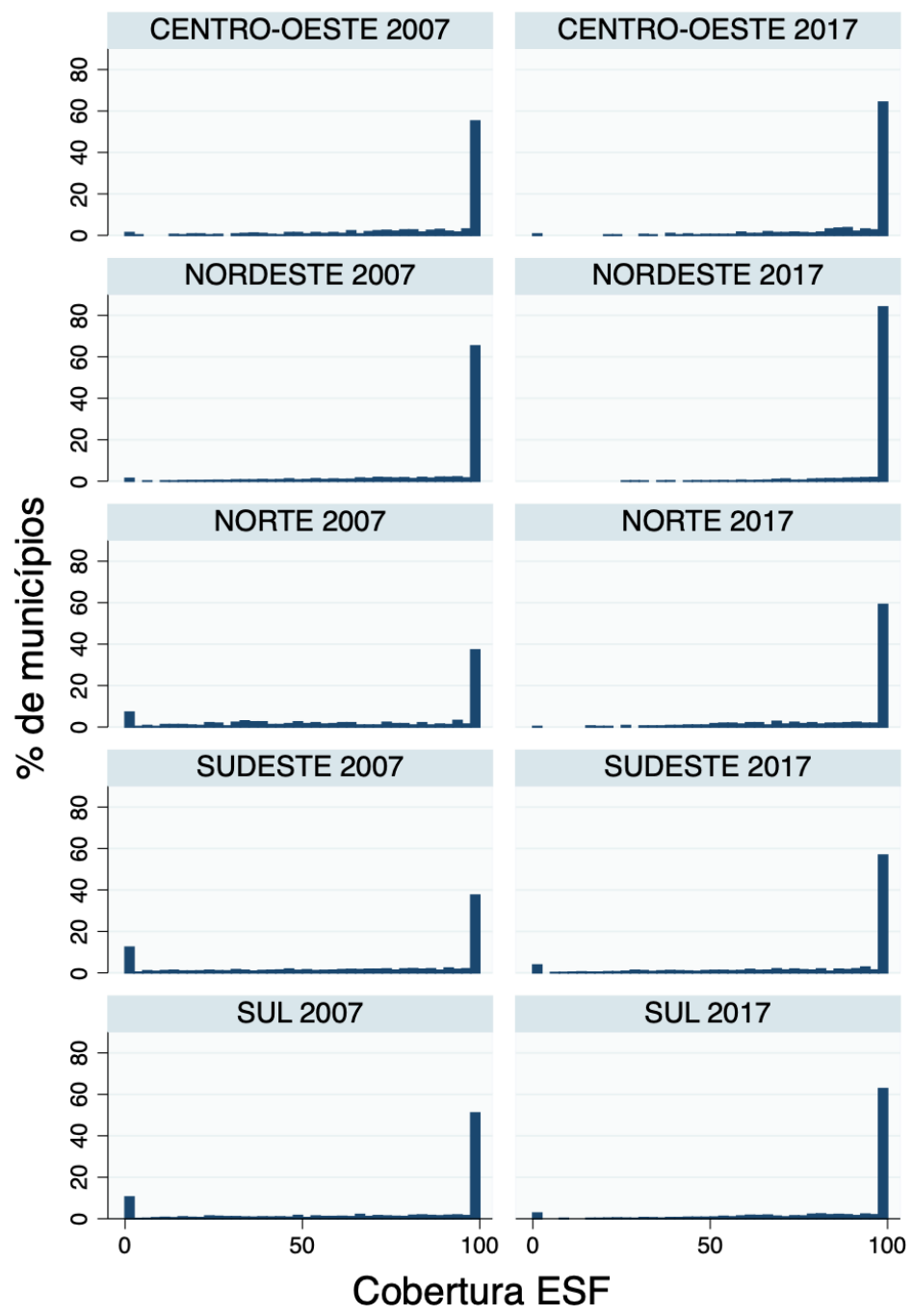

Fonte: Elaboração própria com dados do CNES. 
Em relação ao tamanho do município, é importante notar que o percentual de cobertura municipal do PSF cai proporcionalmente com o aumento da população (Tabela 1). Para ilustrar, em 20072601 municípios brasileiros possuíam menos de $10 \mathrm{mil}$ habitantes e, nestes, a cobertura média do PSF era de $86,95 \%$, passando a $94,98 \%$ em 2017 . No outro extremo, para municípios com mais de 500 mil habitantes, em 2007 a cobertura era de 32,81\%, passando a 44,5\% em 2017. É possível dizer que este resultado, de relação inversa entre a cobertura do PSF e a escala urbana, apresenta um importante caráter equitativo, já destacado por Andrade et al. (2015). O aumento da escala urbana tende a trazer consigo uma elevação da renda e elevação das possibilidades de acesso a saúde, reduzindo, em alguma medida, esta demanda pela atenção primária do PSF.

De outra forma, a cobertura tende a ser maior naquelas localidades de menor renda, com menor acesso aos serviços públicos e privados em saúde. Assim, em uma perspectiva de provisão equitativa de serviços em saúde, localidades com alta renda, com maior percentual de cobertura por planos de saúde, não se apresentariam como prioridades de oferta do PSF. O entendimento é que tal comportamento seria direcionado na focalização da importante política de prover atenção primária, porta de entrada de um sistema gigante, complexo e hierarquizado do SUS, em um cenário de recursos escassos e precariedade.

Tabela 1 - Cobertura populacional do Programa Saúde da Família, por porte do município, Brasil - 2007 e 2017

\begin{tabular}{cccc}
\hline & Ano & $\begin{array}{c}\text { Quantidade } \\
\text { de } \\
\text { Municípios }\end{array}$ & Média \\
\hline $\begin{array}{c}\text { Até } 10 \text { mil } \\
\text { habitantes }\end{array}$ & 2007 & 2601 & $86.95 \%$ \\
\hline $\begin{array}{c}\text { De } \mathbf{1 0} \text { a } \mathbf{5 0} \text { mil } \\
\text { habitantes }\end{array}$ & 2017 & 2450 & $94.98 \%$ \\
\hline $\begin{array}{c}\text { De } \mathbf{5 0} \text { a } \mathbf{1 0 0} \text { mil } \\
\text { habitantes }\end{array}$ & 2017 & 2396 & $72.51 \%$ \\
\hline $\begin{array}{c}\text { De } \mathbf{1 0 0} \text { a } \mathbf{5 0 0} \text { mil } \\
\text { habitantes }\end{array}$ & 2007 & 314 & $51.17 \%$ \\
\hline Mais de 500 mil & 2017 & 355 & $72.25 \%$ \\
\hline habitantes & 2007 & 217 & $37.68 \%$ \\
\hline Total & 2017 & 42 & $55.76 \%$ \\
\hline & 2007 & 5564 & $76.36 \%$ \\
\hline
\end{tabular}

Fonte: Elaboração própria com dados do CNES. 
Diversos fatores podem impactar na adoção e expansão do Programa Saúde da Família em um município, que devem ser compreendidas como importantes estratégias de universalização do acesso à saúde (Andrade et al., 2018b). Além desta perspectiva da cobertura, que ilustra importante evolução no período analisado ao mesmo tempo que sugere focalização em áreas mais demandantes, um importante indicador da qualidade do serviço prestado tende a estar relacionado com o profissional médico da saúde e da comunidade.

Objetivando esta melhoria do serviço prestado, é importante destacar esforços recentes do Governo Federal na direção de ampliar a presença deste médico nas mais diversas localidades. Neste cenário, destaca-se o esforço empenhado via Programa Mais Médicos, com importantes avanços em regulamentação e estruturação para a ampliação da formação do médico da família e da comunidade. Barbosa et al. (2018) indicam que as evidências sobre a atenção primária à saúde são positivas quanto ampliação do acesso e da melhoria da qualidade do serviço prestado, destacando a importância de se avaliar com mais robustez os impactos do PMM.

A Lei no 12.871 de 2013, em seu artigo 4으, estabelece a exigência de que $30 \%$ da carga horária do internato do curso médico ser realizado na atenção primária a saúde e em serviços de Urgência e Emergência do SUS, além da exigência de que esta readequação se dê até 2018. Nesta orientação, pesaria o fato de existir uma correlação direta entre a escolha do médico em trabalhar com atenção primária e a existência de estágios obrigatórios em Medicina da Família em sua graduação, além da importância de experiências longitudinais (GONÇALVES et al., 2009). A proposta é de ampliar o contato do estudante de medicina com o PSF e tentar atrai-lo a trabalhar na atenção primária, apresentando uma visão mais completa e abrangente do funcionamento do sistema único de saúde e da importância da atenção primária.

Outra intervenção governamental, também destacada na Lei no 12.871 de 2013, estaria na estrutura da Residência Médica, que passaria pela obrigatoriedade, a partir de 2018 , da realização de um ano de residência em "Medicina Geral de Família e Comunidade".

Especificamente em relação a residência, Gonçalves et al. (2009) destacam, também, que existe uma correlação entre a fixação dos médicos e o local de realização da residência médica, se comparado ao local da graduação. Para o caso da atenção primária, que demanda significativa capilaridade em sua implementação, o desafio é fomentar o deslocamento do médico às mais distintas localidades, dada que ainda há uma concentração de vagas de residência médica nos grandes centros urbanos e capitais.

Neste sentido, a próxima seção apresenta e discute os avanços na formação do Médico da Família e da Comunidade e sua distribuição no território nacional. Constatada a significativa concentração territorial na sua formação, para uma mais efetiva dispersão deste profissional no território, destacase a necessidade de avançar na interiorização das residências.

\section{FORMAÇÃO PROFISSIONAL E DESCENTRALIZAÇÃO}

A Tabela 2 apresenta a evolução do número de vagas de residência médica no Brasil entre 2014 e 2017. Apesar de o trabalho focar na evolução dos indicadores da atenção primária a saúde entre 2007 e 2017, a Tabela 2 apresenta o resultado de uma expansão planejada pelo Governo Federal e instituída em 2013 (Lei no 12.871).

O principal ponto a se observar na evolução ilustrada pela Tabela 2 é que a expansão do número de vagas para residência em Medicina da Saúde e da Comunidade é substancialmente superior a expansão geral das vagas em residência. Enquanto houve um crescimento de aproximadamente $30 \%$ nas vagas em todas as especialidades, o número de vagas na residência em medicina da família e comunidade foi expandido em aproximadamente 150\% entre 2014-2017. Em todas as regiões a expansão foi maior para este grupo em comparação a expansão geral. Esta constatação permite afirmar sobre o esforço recente de elevação da qualidade da atenção primaria pela melhoria da provisão deste profissional. 
Tabela 2 - Evolução do número vagas de Residência em Medicina da Família e Comunidade R1 autorizadas e Residência em todas as especialidades, por região, Brasil - 2014 à 2017

\begin{tabular}{|c|c|c|c|c|c|c|c|}
\hline Região & $\begin{array}{l}2014 \\
\text { Vagas }\end{array}$ & $\begin{array}{l}\text { Aumento \% } \\
\text { (2014-2015) }\end{array}$ & $\begin{array}{l}2015 \\
\text { Vagas }\end{array}$ & $\begin{array}{l}\text { Aumento \% } \\
(2015-2016)\end{array}$ & $\begin{array}{l}2016 \\
\text { Vagas }\end{array}$ & $\begin{array}{l}\text { Aumento \% } \\
(2016-2017)\end{array}$ & $\begin{array}{l}2017 \\
\text { Vagas }\end{array}$ \\
\hline \multicolumn{8}{|c|}{ Residência em Medicina da Família e Comunidade (R1) } \\
\hline Norte & 109 & 6,42 & 116 & 46,55 & 170 & 2,94 & 175 \\
\hline Nordeste & 268 & 47,01 & 394 & 67,51 & 660 & 6,67 & 704 \\
\hline Sudeste & 587 & 13,12 & 664 & 75,30 & 1.164 & 35,40 & 1.576 \\
\hline Sul & 285 & 8,42 & 309 & 84,79 & 571 & 4,03 & 594 \\
\hline Centro-Oeste & 40 & 30,00 & 52 & 188,46 & 150 & 10,00 & 165 \\
\hline Brasil & 1.289 & 19,08 & 1.535 & 76,87 & 2.715 & 18,38 & 3.214 \\
\hline \multicolumn{8}{|c|}{ Residência em todas as especialidades (R1) } \\
\hline Norte & 717 & 12,13 & 804 & 14,05 & 917 & 7,42 & 985 \\
\hline Nordeste & 2.887 & 10,81 & 3.199 & 12,69 & 3.605 & 2,77 & 3.705 \\
\hline Sudeste & 10.940 & 7,88 & 11.802 & 7,32 & 12.666 & 15,26 & 14.599 \\
\hline Sul & 3.137 & 8,67 & 3.409 & 12,94 & 3.850 & 2,86 & 3.960 \\
\hline Centro-Oeste & 1.272 & 5,50 & 1.342 & 13,93 & 1.529 & 1,90 & 1.558 \\
\hline Brasil & 18.953 & 8,46 & 20.556 & 9,78 & 22.567 & 9,93 & 24.807 \\
\hline
\end{tabular}

Fonte: Elaboração própria com dados SisCNRM.

Por se tratar da elevação da oferta de vagas em residência médica, em resposta a uma legislação criada, reforça-se a importância da atuação governamental como fomentadora da atenção primária a saúde.

O nível de agregação dos dados da Tabela 2 não permite uma melhor análise da capilaridade da formação deste profissional, tendo como foco a discussão relacionada ao vínculo criado e à possibilidade de permanência deste profissional na localidade em que se formou. A Figura 3 apresenta a distribuição no território brasileiro das matrículas em cursos de medicina. Esta distribuição pode ser entendida como uma proxy para as localidades em que são oferecidas as residências, pela elevada correlação entre as escolas de medicina e a oferta de vagas em residência.

A Figura 3 apresenta que houve melhoria na distribuição territorial na formação, porém bastante aquém do necessário para cobrir o território nacional, permanecendo notória a concentração de vagas na região sudeste e nas capitais das UFs. A expansão das vagas e maior capilaridade também foi foco de política pública, com parte da elevação sendo atendida pela expansão do serviço público de educação e uma parcela maior sendo atendida pela iniciativa privada. Silva Júnior e Andrade (2016) apresentam que nos dois anos iniciais do PMM foi autorizada a criação de 5,3 mil novas vagas de graduação em medicina, das quais 
Figura 3 - Número de matrículas em cursos de Medicina, por município, Brasil, 2009 e 2017

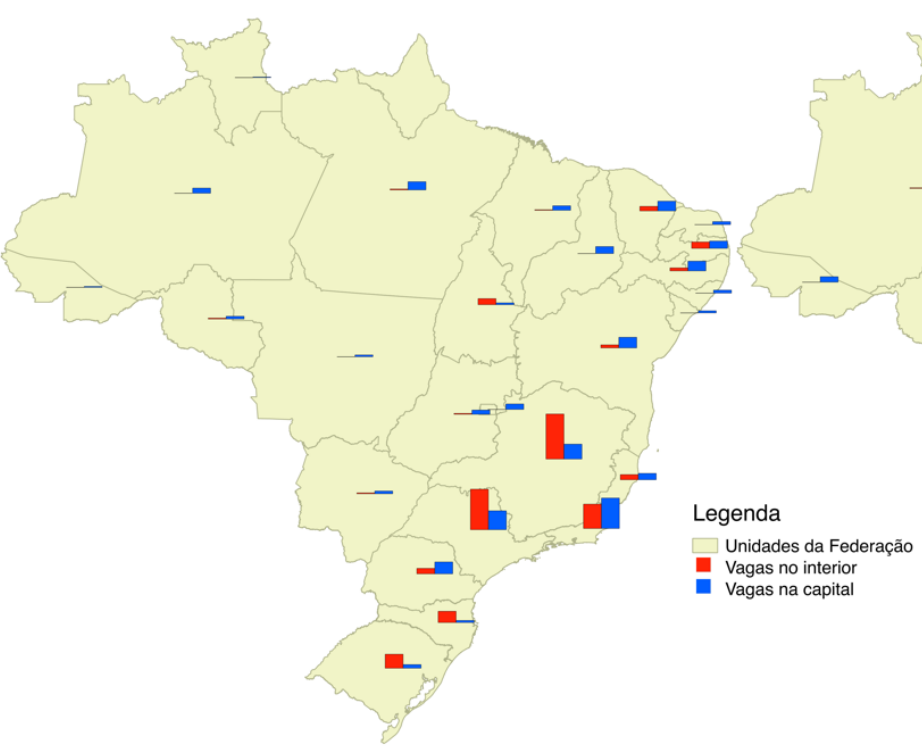

(a) 2009

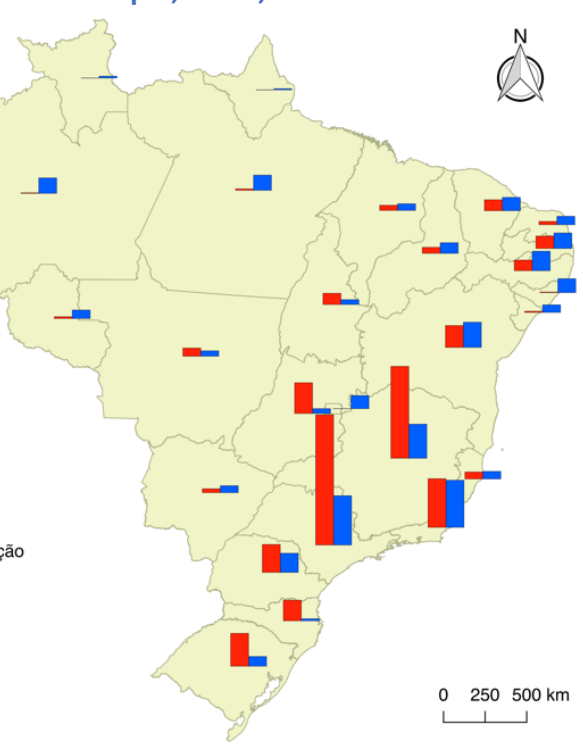

(b)

2017

Fonte: Elaboração própria com dados do Censo do Ensino Superior, INEP, 2009 e 2017.

De qualquer forma, nota-se a necessidade de um esforço por atrair o profissional médico para áreas relativamente distante das áreas em que estes foram formados. Este é um desafio que deve passar pela lógica da gestão de recursos humanos (ANDRADE et al., 2015). A precariedade dos vínculos trabalhistas, a necessidade de atração e retenção destes profissionais para as mais distintas localidades do território nacional, muitas vezes áreas carentes, trazem o desafio de construir capacidade de fazer a gestão deste recurso, normalmente escasso em diversas localidades. Sob esta perspectiva, Barbosa et al. (2018) inferem sobre a diminuição da rotatividade dos profissionais médicos, como uma decorrência do PMM, o que também tende a ser bastante positivo em termos de elevação da qualidade do serviço prestado por este profissional.

Em relação aos profissionais da categoria Médico da Família e da Comunidade, a Figura 4 evidencia que houve melhoria na sua distribuição territorial entre 2007 e 2017, ainda que não se possa inferir sobre avanços na gestão de recursos humanos. Usando o parâmetro de referência de 1 médico para cada 2 mil habitantes (BRASIL, 2015), o mapa apresenta a evolução da cobertura populacional, com o indicador $100 \%$ indicando que o número de profissionais está de acordo com o parâmetro.

Neste sentido, fica demostrado que, apesar da concentração da formação (Figura 3), o País avançou neste período na interiorização deste profissional, levando-os aos mais diversos municípios do território. Isso permite inferir sobre a melhoria na assistência primária a saúde no território nacional. 


\section{Artigos}

Figura 4 - Cobertura populacional de Médicos da Família e da Comunidade, por município, Brasil - 2007 e 2017

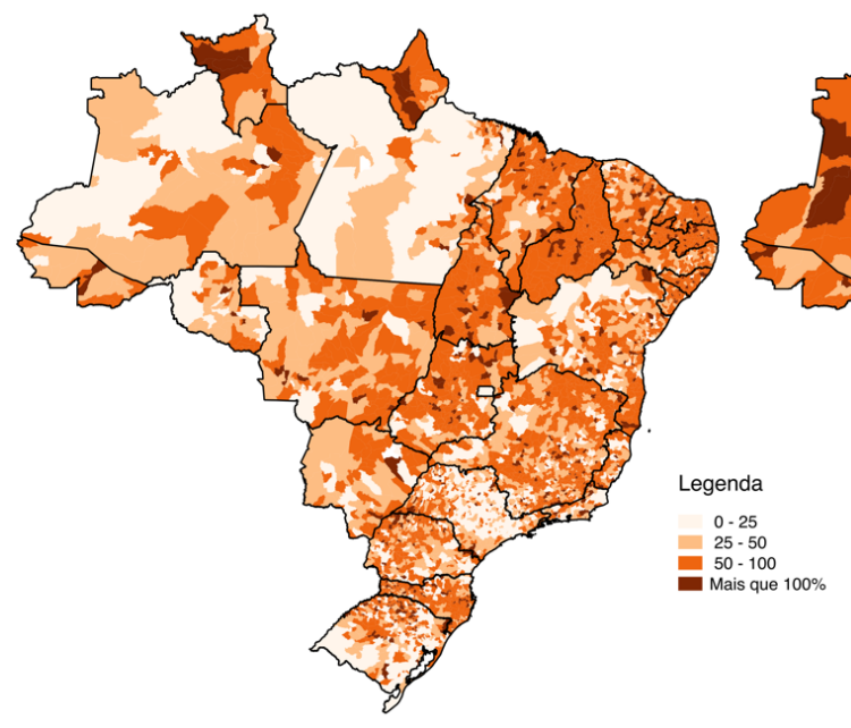

(a) 2007

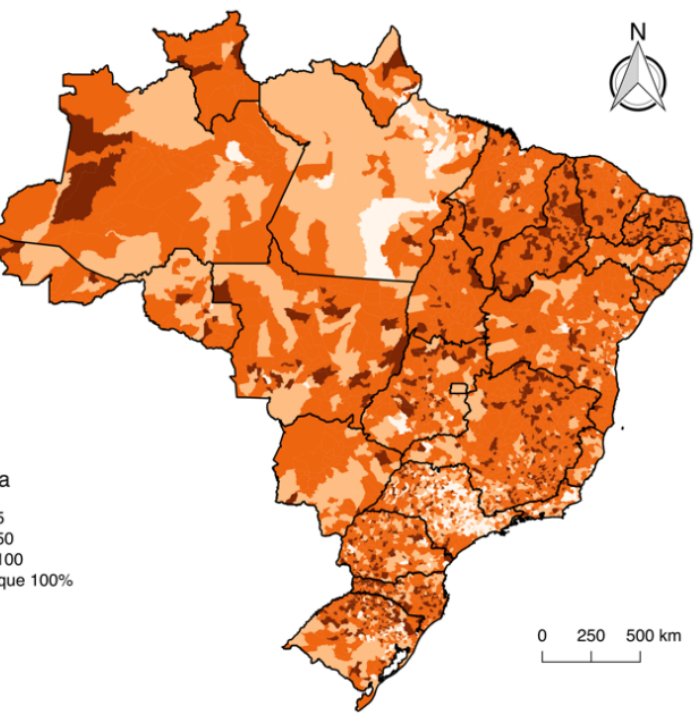

(b) 2017

Fonte: Elaboração própria com dados do CNES.

Também sobre a perspectiva de desconcentração, não mais com foco na distribuição em todo o território, mas na distribuição de acordo com o tamanho do município, a Tabela 3 apresenta a evolução da alocação do Médico da Família e da Comunidade em cinco perfis municipais, definidos pela sua população. Para municípios com até $10 \mathrm{mil}$ habitantes, em 2007 havia aproximadamente 0,37 profissionais para cada 1 mil habitantes, tendo este número passado a aproximadamente 0,46 profissionais em 2017. A cobertura média nacional em 2007 era de 0,30 profissionais, indo a 0,38 em 2017

Também como observado para a cobertura municipal do PSF, na alocação de Médicos da Família e da Comunidade a cobertura também diminui com o aumento da escala urbana. De forma semelhante, a justificativa estaria relacionada a diminuição das possibilidades de acesso a serviços de saúde em municípios menores, o que demandaria uma maior provisão deste profissional em atenção primária.

Tanto para a cobertura do PSF quanto para a presença do profissional médico, fica evidenciado que houve substancial avanço entre 2007 e 2017. Os dados sugerem que o PMM foi de grande importância para os avanços observados no período, em termos do seu alcance territorial. De qualquer forma, Barbosa et al. (2018) chamam a atenção para a necessidade em se avançar na avaliação dos impactos, especificamente do PMM, porém sem negar as melhorias já observadas. 
Tabela 3 - Médicos da Família e da Comunidade (por mil habitantes), por porte do município, Brasil - 2007 e 2017

\begin{tabular}{|c|c|c|c|c|c|}
\hline & Ano & $\begin{array}{c}\text { Quantidade } \\
\text { de Municípios }\end{array}$ & Média & Mínimo & Máximo \\
\hline \multirow{2}{*}{$\begin{array}{l}\text { Até } 10 \text { mil } \\
\text { habitantes }\end{array}$} & 2007 & 2601 & 0.3684 & 0.00 & 1.39 \\
\hline & 2017 & 2450 & 0.4632 & 0.00 & 1.76 \\
\hline \multirow{2}{*}{$\begin{array}{l}\text { De } 10 \text { a } 50 \text { mil } \\
\text { habitantes }\end{array}$} & 2007 & 2396 & 0.2689 & 0.00 & 0.99 \\
\hline & 2017 & 2455 & 0.3446 & 0.00 & 1.43 \\
\hline \multirow{2}{*}{$\begin{array}{c}\text { De } 50 \text { a } 100 \mathrm{mil} \\
\text { habitantes }\end{array}$} & 2007 & 314 & 0.1765 & 0.00 & 0.50 \\
\hline & 2017 & 355 & 0.2466 & 0.00 & 0.56 \\
\hline \multirow{2}{*}{$\begin{array}{l}\text { De } 100 \text { a } 500 \text { mil } \\
\text { habitantes }\end{array}$} & 2007 & 217 & 0.1300 & 0.00 & 0.43 \\
\hline & 2017 & 268 & 0.1933 & 0.00 & 0.46 \\
\hline \multirow{2}{*}{$\begin{array}{l}\text { Mais de } 500 \text { mil } \\
\text { habitantes }\end{array}$} & 2007 & 36 & 0.1158 & 0.00 & 0.31 \\
\hline & 2017 & 42 & 0.1633 & 0.08 & 0.36 \\
\hline \multirow{2}{*}{ Total } & 2007 & 5564 & 0.3038 & 0.00 & 1.39 \\
\hline & 2017 & 5570 & 0.3819 & 0.00 & 1.76 \\
\hline
\end{tabular}

Fonte: Elaboração própria com dados do CNES.

\section{DISCUSSÃO}

Esse trabalho apresentou os significativos avanços do Programa Saúde da Família em relação a sua implementação e cobertura, especificamente na última década. $O$ aumento da cobertura populacional desse programa foi fundamental para atingir a melhora nos índices de saúde geral dessas populações observado no período analisado. Embora tenha sido constatado o avanço do Programa Saúde da Família em relação à sua expansão e aumento de oferta, resultante de uma política pública para prover atenção primária, ainda há um enorme esforço a ser desprendido no provimento de uma assistência primária cada vez mais eficiente e longitudinal.

Dentre os entraves para a melhora na qualidade da atenção primária, destacamos a necessidade da oferta de um profissional cada vez mais qualificado e especializado: o médico da família e comunidade. Foi constatada a expansão dos cursos de medicina, assim como das vagas de residência em medicina da família e comunidade nos últimos anos, este último, derivado do PMM, que fomenta a formação de recursos humanos para diminuir a carência de médicos nas regiões com menor poder de atração destes profissionais. Não obstante, essa expansão foi concentrada na região Sudeste e em algumas capitais estaduais, sem ser observado uma interiorização da formação desse especialista. Cabe, porém, investigar em que medida as políticas desenhadas no PMM estão sendo cumpridas como, por exemplo, na obrigatoriedade de um ano de residência em Medicina Geral de Família e Comunidade, com prazo para adequação no fim de 2018.

Somado ao desafio de formação e atração desse profissional, tem-se a dificuldade em reter esse 
especialista em um município. Por tais razões anteriormente elencadas, destacamos a necessidade da modernização na gestão de recursos humanos como, por exemplo, estabelecendo processos de modernização dos vínculos trabalhistas e avançando na capacitação desses profissionais com intuito de atrair e fixar esses trabalhadores em áreas carentes, para potencializar o papel equitativo do Programa Saúde da Família.

\section{REFERÊNCIAS BIBLIOGRÁFICAS}

(1) BARBOSA, Allan Claudius Queiroz et al. Programa Mais Médicos: como avaliar o impacto de uma abordagem inovadora para superação de iniquidades em recursos humanos. Revista Panamericana de Salud Pública, v. 42, p. e185, 2018.

(2) ANDRADE, Mônica Viegas et al. A equidade na cobertura da Estratégia Saúde da Família em Minas Gerais, Brasil. Cadernos de Saúde Pública, v. 31, p. 1175-1187, 2015.

(3) ANDRADE, Mônica Viegas et al. Family health strategy and equity in prenatal care: a population based cross-sectional study in Minas Gerais, Brazil. International journal for equity in health, v. 16, n. 1, p. 24, 2017.

(4) ANDRADE, Mônica Viegas et al. Brazil's Family Health Strategy: factors associated with programme uptake and coverage expansion over 15 years (1998-2012). Health policy and planning, v. 33 , n. 3, p. 368-380, 2018a.

(5) ANDRADE, Mônica Viegas et al. Transition to universal primary health care coverage in Brazil: analysis of uptake and expansion patterns of Brazil's Family Health Strategy (1998-2012). PloS one, v. 13, n. 8, p. e0201723, 2018b.

(6) BITTENCOURT, Roberto José. A superlotação dos serviços de emergência hospitalar como evidência de baixa efetividade organizacional. Tese (Doutorado) - Fundação Oswaldo Cruz, Rio de Janeiro, fev 2010.

(7) BORLINI, Leandra Maria. Há pedras no caminho do SUS: os impactos do neoliberalismo na saúde no Brasil. Textos e Contextos, Porto Alegre, v. 9, n. 2, p. 321-333, ago 2010.

(8) BRASIL. Ministério da Saúde. Portaria no 1.631/GM de 30 de julho de 2015. Brasília, 2015.

(9) CAMPOS, Gastão Wagner de Sousa; PEREIRA JÚNIOR, Nilton. A atenção primária e o Programa Mais Médicos do Sistema Único de Saúde: conquistas e limites. Ciência \& Saúde Coletiva, v. 21, p. 2655-2663, 2016.

(10) KRINGOS, Dionne S. et al. Europe's strong primary care systems are linked to better population health but also to higher health spending. Health affairs, v. 32, n. 4, p. 686-694, 2013.

(11) LENTSCK, Maicon Henrique; KLUTHCOVSKY, Ana Cláudia Garabeli Cavalli; KLUTHCOVSKY, Fábio Aragão. Avaliação do Programa Saúde da Família: uma revisão. Ciência \& Saúde Coletiva, v. 15, 2010, p. 3455-3466.

(12) MACINKO, James; STARFIELD, Barbara; SHI, Leiyu. The contribution of primary care systems to health outcomes within Organization for Economic Cooperation and Development (OECD) countries, 1970-1998. Health services research, v. 38, n. 3, p. 831-865, 2003.

(13) OLIVEIRA, Felipe Proenço de et al. Mais Médicos: um programa brasileiro em uma perspectiva internacional. Interface-Comunicação, Saúde, Educação, v. 19, p. 623-634, 2015.

(14) SANTOS, Isabela Soares et al. Mix públicoprivado no sistema de saúde brasileiro: realidade e futuro do SUS. In: FUNDAÇÃO OSWALDO CRUZ. A saúde no Brasil em 2030 - prospecção estratégica do sistema de saúde brasileiro: estrutura do financiamento e do gasto setorial [online]. Rio de Janeiro: $\quad$ Fiocruz/Ipea/Ministério da Saúde/Secretaria de Assuntos Estratégicos da Presidência da República, 2013. Vol. 4. pp. 73-131. ISBN 978-85-8110-018-0.

(15) SANTOS, Leonor Maria Pacheco; COSTA, Ana Maria; GIRARDI, Sábado Nicolau. Programa Mais Médicos: uma ação efetiva para reduzir iniquidades em saúde. Ciência \& Saúde Coletiva, v. 20, p. 3547-3552, 2015. 


\section{Artigos}

(16) SILVA JUNIOR, Aluísio Gomes da; ANDRADE, Henrique Sater de. Formação médica no Programa Mais Médicos: alguns riscos. Ciência \& Saúde Coletiva, v. 21, p. 2670-2671, 2016.

\section{NOTAS DE RODAPÉ}

Um dos objetivos principais do Programa Mais Médicos, implementado em julho de 2013, é reduzir as iniquidades ao acesso aos serviços de saúde em áreas vulneráveis dos municípios brasileiros. Para tal, ele foi estruturado em três eixos: (i) melhoria da infraestrutura das unidades básicas de saúde; (ii) ampliação e reforma educacional dos cursos de graduação em medicina e residência médica; e (iii) o provimento emergencial de médicos em áreas com baixa oferta. Para maiores informações sobre o Programa Mais Médicos (PMM) e seus impactos ver
Oliveira et al. (2015), Santos et al. (2015), Campos e Pereira Júnior (2016) e Silva Júnior e Andrade (2016).

\section{ABSTRACT}

Since the early 1990s Brazilian health system concentrated efforts on preventive healthcare along with continuous monitoring of the population needs. It has been observed a major extension of the Family Health Strategy across Brazilian territory with significant interiorization, especially in the Northeast and the Midwest regions, as well as in the North of Minas Gerais. It has also been observed an increase in the number of undergraduate vacancies as well as in the number of family and community medicine residency spots, although strongly concentrated in the Southeast region and state capitals. Besides, we stress the need to modernize human resources management as means to draw and retain professionals in the areas most in need, fostering the equalization of the Family Health Strategy.

Keywords: saúde da família, mais médicos, spacial distribution. 